\title{
High-resolution X-ray spectroscopy of the low and high states of the Seyfert 1 galaxy NGC 4051 with Chandra LETGS
}

\author{
K. C. Steenbrugge ${ }^{1}$, M. Feňovčík ${ }^{2}$, J. S. Kaastra ${ }^{2,3}$, E. Costantini ${ }^{2}$, and F. Verbunt ${ }^{3}$ \\ 1 St John's College Research Centre, University of Oxford, Oxford, OX1 3JP, UK \\ SRON Netherlands Institute for Space Research, Sorbonnelaan 2, 3584 CA Utrecht, The Netherlands \\ Astronomical Institute, Utrecht University, PO Box 80000, 3508 TA Utrecht, The Netherlands
}

Received 18 June 2008 / Accepted 15 January 2009

\section{ABSTRACT}

\begin{abstract}
Context. With the new generation of high-resolution X-ray spectrometers the understanding of warm absorbers in active galactic nuclei has improved considerably. However, the important questions regarding the distance and structure of the photoionised wind remain unsolved.

Aims. To constrain the distance of the photoionised wind, we study the variability of the continuum, absorption, and, emission in one of the brightest and most variable low-luminosity AGN: the narrow-line Seyfert galaxy NGC 4051.

Methods. We analyse two observations taken with the Low Energy Transmission Grating Spectrometer of Chandra. We investigated the spectral response to a sudden flux decrease by a factor of 5 , which occurred during the second observation.

Results. We detect a highly ionised absorption component with an outflow velocity of $-4670 \mathrm{~km} \mathrm{~s}^{-1}$, one of the highest outflow velocity components observed in a Seyfert 1 galaxy. Furthermore, this is one of the only observations whereby the X-ray observed absorption component is unaccompanied by a corresponding UV absorption component with the same outflow velocity. The spectra contain a relativistic $\mathrm{O}$ VIII Ly $\alpha$ line with properties similar to those determined for this source with XMM-Newton, and four absorption components spanning a range in ionisation parameter $\xi$ between 0.07 and 3.19 (log values, and units of $10^{-9} \mathrm{~W} \mathrm{~m}$ ). An emission component producing radiative recombination continua of $\mathrm{C} \mathrm{VI}$ and $\mathrm{C} \mathrm{V}$ appears during the low state. The black body temperature decreases with the drop in flux observed in the second observation.

Conclusions. For all absorber components we exclude that the ionisation parameter linearly responded to the decrease in flux by a factor of 5. The variability of the absorber suggest that at least three out of four detected components are located in the range $0.02-1 \mathrm{pc}$. For one component we only have a lower limit of $0.3 \mathrm{pc}$. These distances are different from earlier suggestions.
\end{abstract}

Key words. galaxies: Seyfert - quasars: individual: NGC 4051 - galaxies: active - X-rays: galaxies

\section{Introduction}

The stupendous amount of energy emitted by an active galactic nucleus (AGN) is released by gas that flows towards a supermassive black hole in the centre of a galaxy, presumably via an accretion disc. The average energy of the emitted radiation increases with decreasing distance to the super-massive black hole, thus X-rays provide the best probe of the gas flow in the immediate surroundings of the black hole. From the presence of jets, as well as from blue-shifted ultraviolet absorption lines, we learn that there is gas flowing away from the black hole, in addition to the flow towards it. With the availability of X-ray spectrographs onboard Chandra and XMM-Newton, we can study the lines emitted in the X-ray band from these outflows close to the black hole. The presence of multiple absorption line systems that differ in their level of ionisation or in their outflow velocity constrains the geometry of the outflow (for example NGC 3783, Netzer et al. 2003; NGC 5548, Steenbrugge et al. 2005).

In this paper we describe the X-ray spectra of the narrowline Seyfert 1 galaxy NGC 4051, obtained with the Low-Energy Transmission Grating (LETG) on board of the Chandra satellite. Emission from the nucleus of NGC 4051 was detected by Hubble (1926), and the galaxy is one of the 12 discussed in the seminal paper by Seyfert (1943). As a member of the Ursa Major cluster (Tully et al. 1996), it has a distance of $18.6 \mathrm{Mpc}$ (Tully \& Pierce 2000) and a heliocentric velocity of $700 \mathrm{~km} \mathrm{~s}^{-1}$
(Verheijen \& Sancisi 2001). NGC 4051 is a relatively bright optical object, with $V \simeq 13.5$. The soft excess observed with the Reflection Grating spectrometers (RGS) onboard XMM-Newton is not described well by a multi-temperature disc: Ogle et al. (2004) concludes that the soft excess is due to emission from relativistically broadened $\mathrm{O}$ VIII emission lines and its related radiative recombination continuum (RRC). However, Pounds et al. (2004) fitted the EPIC data of the same observation satisfactorily with a power law plus two black body components.

Two absorption line systems, at $-2340 \pm 130$ and $-600 \pm$ $130 \mathrm{~km} \mathrm{~s}^{-1}$, were detected in the X-ray spectrum with the High Energy Transmission Grating of Chandra (Collinge et al. 2001). Nine absorption systems with a small range in outflow velocity are detected in the ultraviolet with the Space Telescope Imaging Spectrograph (STIS) and the Far Ultraviolet Spectroscopic Explorer (FUSE). One of the absorbers observed in the UV may coincide with the $-600 \pm 130 \mathrm{~km} \mathrm{~s}^{-1} \mathrm{X}$-ray absorption system, all the others have smaller outflow velocities (Collinge et al. 2001; Kaspi et al. 2004).

In a preliminary analysis of our first LETGS spectrum, van der Meer et al. (2003) did not detect the $-2340 \mathrm{~km} \mathrm{~s}^{-1}$ velocity component, but found indications for the presence of an outflow component at an even higher velocity of $-4500 \mathrm{~km} \mathrm{~s}^{-1}$. The second observation, taken when NGC 4051 was in a much higher flux state, allows us to investigate the ionised outflow both 

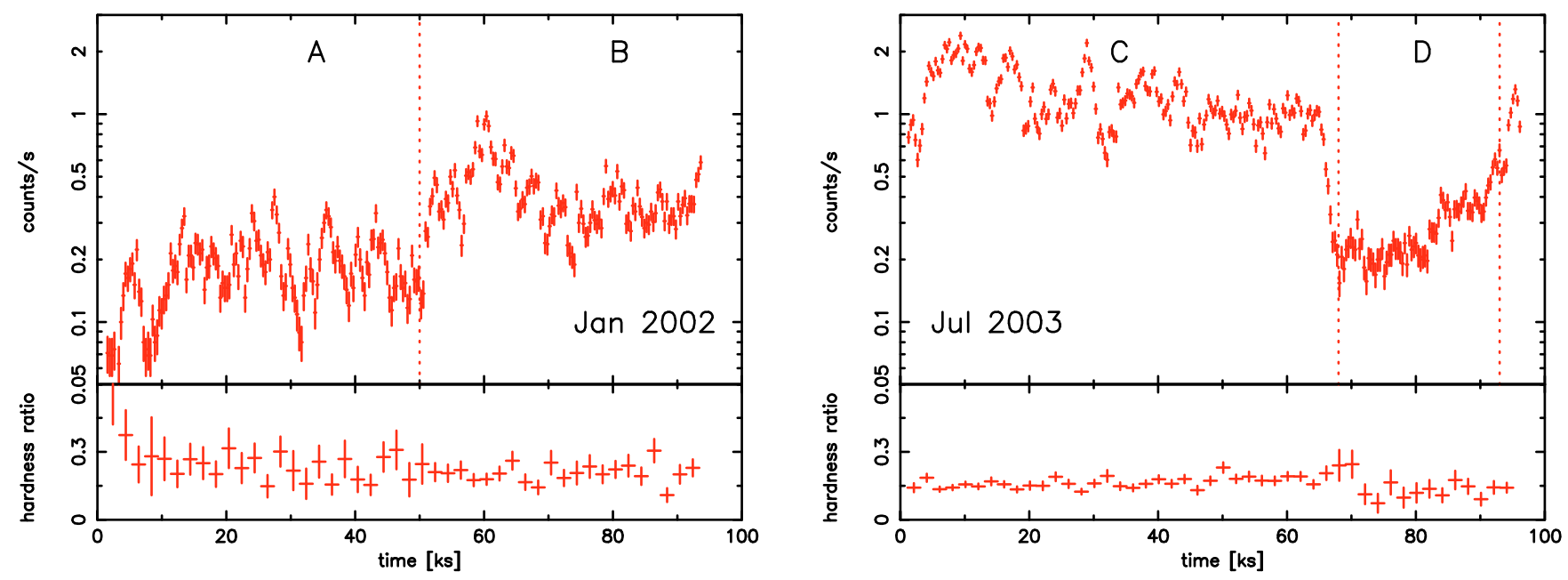

Fig. 1. The light curve in the zeroth spectral order (top panels) and hardness ratio (bottom panels) for the observations of Jan. 2002 and July 2003. The hardness ratio is defined as the ratio of the 2-8 and 8-32 A counts. The top panel has a bin size of $350 \mathrm{~s}$ and the bottom panel has a bin size of 2000 s. Dotted lines denote the different flux states and define the intervals used in creating the four spectra.

in terms of outflow velocity and ionisation structure. In this paper we report the analysis of both observations with the LETGS.

\section{Observations and data reduction}

In this paper we describe the two observations of NGC 4051 taken with the LETGS (Brinkman et al. 2000) onboard of Chandra. The detector used was the High-Resolution Camera (HRC-S). The observations span the time periods December 31, 2001 17:40:41 to January 1, 2002 19:51:46 and July 23, 2003 $00: 34: 15$ to July 24 03:24:43, and have a net exposure time of 94.2 and $96.6 \mathrm{ks}$, respectively. Henceforth we will we refer to these observations as the Jan. 2002 and Jul. 2003 observations. The data reduction method is described in detail by Kaastra et al. (2002). After event selection we produced background subtracted spectra and light curves. As the HRC-S detector has limited energy resolution, higher spectral orders cannot be separated in the data but are taken into account during spectral fitting. We only use spectra between $2 \AA$ and $80 \AA$, as the Galactic absorption attenuates the spectrum strongly above $\sim 80 \AA$.

\section{Variability}

Figure 1 shows the light curves for each observation. For the Jan. 2002 observation, the average brightness of the source increased noticeably in the second half of the exposure. The source was brighter still during the first $\sim 65 \mathrm{ks}$ of the Jul. 2003 observation and then dropped in about $3 \mathrm{ks}$ to a lower flux level, which is similar to the flux level of the second half of the first observation. The flux recovered gradually to the high level of the first half of the observation during the final $20 \mathrm{ks}$. On top of these long-term variations we also detect variability with smaller, but significant, amplitude on timescales of a few thousand seconds. These rapid variations have smaller amplitudes after the rapid flux decrease of the Jul. 2003 observation. In the analysis below we will discuss the spectra at different flux levels, defined by the lightcurve of Fig. 1: A and B are the first and second half of the Jan. 2002 observation; C and D are the first and second part of the Jul. 2003 observation.

In Fig. 1 we also show the hardness ratio, defined as the ratio between the count rates in the $2-8 \AA$ and $8-32 \AA$ band passes.
The hardness variation between different flux levels is small, with $H R=0.239 \pm 0.14,0.203 \pm 0.008,0.161 \pm 0.003,0.134 \pm$ 0.021 for intervals $\mathrm{A}-\mathrm{D}$, respectively with their $1 \sigma$ error.

\section{Spectral data analysis and results}

From the above (Fig. 1) it is clear that NGC 4051 shows significant luminosity variability during our observations. As the level of the variations is quite strong (e.g., a factor of five in flux between parts C and D of the July 2003 observation), it is not useful to analyse the integrated spectra as a whole, but we will rather analyse spectra A-D separately, with a focus on the spectral differences between these parts. Statistics prohibit us to do spectral analysis down to the shortest variability timescales of less than $1000 \mathrm{~s}$.

For all spectral fitting we used the SPEX code developed and regularly updated with new models and atomic data at SRON (Kaastra et al. 1996) ${ }^{1}$. As the flux is low in some of the spectra, we cannot use $\chi^{2}$ minimisation, instead we minimise the $C$-statistic. Note that that the uncertainty in absolute calibration for the Chandra LETGS is $13 \mathrm{~m} \AA$. Therefore a velocity difference smaller than $\sim 200 \mathrm{~km} \mathrm{~s}^{-1}$ between spectra A/B and C/D is unlikely due to an intrinsic velocity shift.

For our spectral model we included different components: a broad-band continuum, narrow and broad emission features, and photoionised absorption. The need for these components and the resulting parameters will be discussed separately.

Noting that spectrum $\mathrm{C}$ has the highest signal-to-noise of the four spectra, we decided to fit this spectrum first and then use the best fit model as a template for fitting spectrum B. The signal-tonoise ratios for spectra A and D are poor, therefore we only left a limited number of parameters free when fitting these spectra.

The best fit parameters for our spectral model are listed in Table 1. All models were corrected for Galactic absorption with a column density of $1.31 \times 10^{24} \mathrm{~m}^{-2}$ (Elvis et al. 1989) and for the cosmological redshift (including peculiar motion) of $700 \mathrm{~km} \mathrm{~s}^{-1}$. All errors correspond to $\Delta C=1$ (68\% confidence). The best fit values for the $C$-statistics are 2700 (2476), 2658 (2466), 2738 (2599), and 2870 (2610) for components A-D; the numbers in brackets are the degrees of

1 http://www.sron.nl/spex 
Table 1. The best fit parameters for the different spectral components for the four spectra. All wavelengths and velocities refer to the rest frame of NGC 4051. Numbers in square brackets are calculated from the fit parameters; numbers in round brackets were kept fixed during the fit.

\begin{tabular}{|c|c|c|c|c|}
\hline & $A$ & $B$ & $\bar{C}$ & $\bar{D}$ \\
\hline \multicolumn{5}{|l|}{$\begin{array}{l}\text { Continuum } \\
\text { - power law }\end{array}$} \\
\hline Flux $(2-10 \mathrm{keV})^{a}$ & $0.83 \pm 0.07$ & $1.36 \pm 0.08$ & $2.93 \pm 0.09$ & $0.48 \pm 0.05$ \\
\hline $\begin{array}{l}\text { Photon index } \Gamma \\
\text { - modified black body }\end{array}$ & $1.65 \pm 0.07$ & $2.00 \pm 0.05$ & $2.24 \pm 0.02$ & $2.44 \pm 0.05$ \\
\hline Flux $(0.2-10 \mathrm{keV})^{a}$ & $0.49 \pm 0.08$ & $0.66 \pm 0.14$ & $2.0 \pm 0.2$ & $0.37 \pm 0.09$ \\
\hline$k T(\mathrm{eV})$ & $95 \pm 6$ & $96 \pm 8$ & $143 \pm 4$ & $90 \pm 5$ \\
\hline \multicolumn{5}{|c|}{$\begin{array}{l}\text { Absorption components } \\
\text { - warm absorber } 1\end{array}$} \\
\hline$N_{\mathrm{H}}\left(10^{25} \mathrm{~m}^{-2}\right)$ & - & - & $0.12 \pm 0.04$ & $(0.12)$ \\
\hline $\log \xi\left(10^{-9} \mathrm{~W} \mathrm{~m}\right)$ & - & - & $0.07 \pm 0.13$ & $0.3 \pm 0.2$ \\
\hline$v_{\text {turb }}\left(\mathrm{km} \mathrm{s}^{-1}\right)$ & - & - & $300 \pm 60$ & $(300)$ \\
\hline$v_{\text {out }}\left(\mathrm{km} \mathrm{s}^{-1}\right)$ & - & - & $-210 \pm 70$ & $(-210)$ \\
\hline \multicolumn{5}{|l|}{ - warm absorber 2} \\
\hline$N_{\mathrm{H}}\left(10^{25} \mathrm{~m}^{-2}\right)$ & $(0.17)$ & $0.17 \pm 0.05$ & $0.29 \pm 0.05$ & $(0.29)$ \\
\hline $\log \xi\left(10^{-9} \mathrm{~W} \mathrm{~m}\right)$ & $0.95 \pm 0.25$ & $0.49 \pm 0.20$ & $0.87 \pm 0.09$ & $0.52 \pm 0.13$ \\
\hline$v_{\text {turb }}\left(\mathrm{km} \mathrm{s}^{-1}\right)$ & (230) & $230 \pm 120$ & $110 \pm 20$ & (110) \\
\hline$v_{\text {out }}\left(\mathrm{km} \mathrm{s}^{-1}\right)$ & $(-330)$ & $-330 \pm 140$ & $-200 \pm 30$ & $(-200)$ \\
\hline \multicolumn{5}{|l|}{ - warm absorber 3} \\
\hline$N_{\mathrm{H}}\left(10^{25} \mathrm{~m}^{-2}\right)$ & $(0.6)$ & $0.6 \pm 0.3$ & $0.8 \pm 0.4$ & $(0.8)$ \\
\hline $\log \xi\left(10^{-9} \mathrm{~W} \mathrm{~m}\right)$ & $2.23 \pm 0.17$ & $1.98 \pm 0.22$ & $2.32 \pm 0.13$ & $2.4_{-0.2}^{+0.6}$ \\
\hline$v_{\text {turb }}\left(\mathrm{km} \mathrm{s}^{-1}\right)$ & $(100)$ & $100 \pm 50$ & $90 \pm 30$ & $(90)$ \\
\hline$v_{\text {out }}\left(\mathrm{km} \mathrm{s}^{-1}\right)$ & $(-610)$ & $-610 \pm 110$ & $-580 \pm 50$ & $(-580)$ \\
\hline \multicolumn{5}{|l|}{ - warm absorber 4} \\
\hline$N_{\mathrm{H}}\left(10^{25} \mathrm{~m}^{-2}\right)$ & (9) & $9 \pm 5$ & $20 \pm 10$ & (20) \\
\hline $\log \xi\left(10^{-9} \mathrm{~W} \mathrm{~m}\right)$ & $3.06 \pm 0.10$ & $2.92 \pm 0.17$ & $3.19 \pm 0.09$ & $3.2 \pm 0.2$ \\
\hline$v_{\text {turb }}\left(\mathrm{km} \mathrm{s}^{-1}\right)$ & $(120)$ & $120 \pm 80$ & $19 \pm 13$ & (19) \\
\hline$v_{\text {out }}\left(\mathrm{km} \mathrm{s}^{-1}\right)$ & $(-4590)$ & $-4590 \pm 180$ & $-4670 \pm 150$ & $(-4670)$ \\
\hline \multicolumn{5}{|c|}{$\begin{array}{l}\text { Emission lines } \\
\text { - O VIII Ly } \alpha(\text { relativistic) })^{b, c}\end{array}$} \\
\hline $\begin{array}{l}\text { Flux }^{d} \\
q \text { (index Laor profile) }\end{array}$ & $\begin{array}{c}12 \pm 2 \\
(5.2)\end{array}$ & $\begin{array}{c}22 \pm 5 \\
5.2 \pm 0.5\end{array}$ & $\begin{array}{c}6.8 \pm 1.5 \\
2.06 \pm 0.26\end{array}$ & $\begin{array}{l}3.5 \pm 1.1 \\
(2.06)\end{array}$ \\
\hline$F W H M(\AA)$ & [10] & [10] & [3.0] & {$[3.0]$} \\
\hline \multicolumn{5}{|l|}{ - O VIII $L y \alpha(B L R)^{b}$} \\
\hline Flux $^{d}$ & $0.1 \pm 0.2$ & $0.0 \pm 0.2$ & $1.8 \pm 0.6$ & $0.8 \pm 0.4$ \\
\hline \multicolumn{4}{|l|}{ - O VII triplet (BLR) } & $(0.45)$ \\
\hline Flux $^{d}$ & $1.4 \pm 0.6$ & $0.0 \pm 0.7$ & $6.2 \pm 1.4$ & $2.2 \pm 1.2$ \\
\hline$F W H M(\AA)$ & $(1.7)$ & (1.7) & $1.7 \pm 0.4$ & $(1.7)$ \\
\hline$\lambda(\AA)$ & $(22.11)$ & $(22.11)$ & $22.11 \pm 0.13$ & $(22.11)$ \\
\hline \multicolumn{5}{|c|}{ - O VII forbidden (narrow) } \\
\hline Flux $^{d}$ & $0.8 \pm 0.2$ & $0.9 \pm 0.2$ & $1.0 \pm 0.2$ & $1.3 \pm 0.3$ \\
\hline$\lambda(\AA)$ & $22.087 \pm 0.005$ & $22.081 \pm 0.006$ & $22.090 \pm 0.009$ & (22.090) \\
\hline \multicolumn{5}{|l|}{ - C VI $\operatorname{Ly} \alpha(B L R)^{e}$} \\
\hline Flux $^{d}$ & $0.6 \pm 0.4$ & $1.0 \pm 0.5$ & $2.4 \pm 0.8$ & $3.1 \pm 0.7$ \\
\hline$F W H M(\AA)$ & $(0.36)$ & $(0.36)$ & $0.36 \pm 0.13$ & $(0.36)$ \\
\hline
\end{tabular}

${ }^{a}$ Absorption-corrected flux in $10^{-14} \mathrm{~W} \mathrm{~m}^{-2}$; $^{b}$ wavelength is frozen to the rest wavelength of $18.967 \AA$ (for O VIII Ly $\alpha$ ); ${ }^{c}$ and an inclination angle of $48^{\circ} ;{ }^{d}$ absorption-corrected photon flux in photons $\mathrm{m}^{-2} \mathrm{~s}^{-1}$; ${ }^{e}$ wavelength is frozen to the rest wavelength of $33.736 \AA$.

freedom. In Table 2 we show the increase $\Delta C$ of the $C$-statistic when a given spectral component is omitted from the model (and re-fitting the spectrum; the omission of a given component can sometimes be partly compensated by adjusting the parameters of the other components). We did this for spectra B and C, the best quality spectra.

Finally, in spectrum D we afterwards added a spectral component fitting the radiative recombination continua (RRCs). Only the $\mathrm{C} \mathrm{V}$ and $\mathrm{C}$ VI RRCs are present, and can be identified by eye in Fig. 2. The temperature and emission measures $n_{\mathrm{e}} n_{\mathrm{X}} V$ with $V$ the emitting volume, $n_{\mathrm{e}}$ the electron density, $n_{\mathrm{X}}$ the density of the recombining ion $\mathrm{X}$ giving rise to the observed transitions are given in Table 3.

\subsection{Continuum}

In spectrum $\mathrm{C}$ the continuum cannot be fitted with only a power law. The soft excess can be modelled by adding a black body modified by coherent Compton scattering (Kaastra \& Barr 1989). Examining all spectra, we find that the power-law photon index becomes softer as time progresses, and is thus not a simple function of luminosity. The modified black body 
Table 2. Significances of the different spectral components. We list the increase in the $C$-statistic when the component is omitted from the model and the spectrum is refitted.

\begin{tabular}{lcc}
\hline \hline Component & Spectrum B & Spectrum C \\
\hline Modified black body & 7.36 & 88.97 \\
rel. O VIII & 16.84 & 11.32 \\
BLR O VIII & 0 & 16.69 \\
BLR O VII & 0 & 31.49 \\
narrow O VII forbidden & 36.08 & 24.60 \\
BLR C VI & 4.07 & 12.01 \\
warm absorber 1 & - & 14.53 \\
warm absorber 2 & 12.74 & 17.83 \\
warm absorber 3 & 12.75 & 14.55 \\
warm absorber 4 & 45.19 & 32.89 \\
\hline
\end{tabular}

temperature seems to track the luminosity variations; the temperature is highest for the high state and drops back to a lower value after the drop in flux just before the start of spectrum D.

\subsection{The ionised absorber}

Thanks to the excellent spectral resolution of the LETG instrument we can study the warm absorber in detail. We fitted the warm absorber with the xabs model in SPEX. In this model, the ionic column densities are not independent quantities, but are linked via the ionisation parameter $\xi=L / n r^{2}$, where $L$ is the source luminosity, $n$ the hydrogen density and $r$ is the distance from the ionising source. For the spectral energy distribution of the ionising source we used the standard one included in SPEX (see dot-dash line of Fig. 1 in Steenbrugge et al. 2005). In addition to the ionisation parameter, the fit parameters are the hydrogen column density of the absorber $N_{\mathrm{H}}$, the outflow velocity $v_{\text {out }}$, and the turbulent velocity $v_{\text {turb }}$. The advantage of the $x a b s$ model is that all relevant ions are taken into account including also the ones with the weakest absorption features. In the fit the abundances were left frozen to the solar abundances given by Anders \& Grevesse (1989).

Table 1 lists the best fit values for the four different spectra. To adequately describe the warm absorber in spectrum $\mathrm{C}$ we need 4 components. The ionisation parameters range between 0.07 and 3.19 (logarithmic values in $10^{-9} \mathrm{~W} \mathrm{~m}$, used throughout this paper). Adding a 5 th absorption component for spectrum $\mathrm{C}$ does not significantly improve the fit, namely $\Delta C$ decreases by only 1.91. Figure 3 compares the best fit model with 3 and 4 absorption components and Table 4 gives the best fit parameters for the three-absorber-component fit to spectrum $\mathrm{C}$. The fit with 4 warm absorber components better fits the $\mathrm{O} V$ and $\mathrm{O}$ IV absorption lines. In the remaining three spectra due to the lower signal to noise ratios, not all four absorption components are detected.

For spectrum B, we need three components. By comparing the parameters of these components to the parameters of the components for spectrum $\mathrm{C}$, we find that component 1 , which has the smallest hydrogen column density, is lacking in spectrum B.

The statistical quality of spectra A and D are much poorer; in fitting these spectra, we fixed several parameters to the best-fit values obtained for spectra $\mathrm{B}$ and $\mathrm{C}$, respectively. It is quite reasonable to keep the outflow and turbulent velocity constant between observations $\mathrm{A} / \mathrm{B}$ and also between $\mathrm{C} / \mathrm{D}$. When we leave both the column density and the ionisation parameter free in spectrum A, only component 2 is marginally detected. These non-detections do not imply that the warm absorber has disappeared, but merely that the statistical quality of spectra A and D are insufficient to determine its parameters with accuracy. For this reason, we make the additional assumption that the column density of the absorber does not change. Any changes however, are likely to be in the ionization parameter (that is, if it can respond fast enough to the continuum flux variations).

Comparing spectra $\mathrm{C}$ and $\mathrm{D}$, only for component 2 a $2.3 \sigma$ drop in ionisation parameter is found; the other components are consistent with no change in the warm absorber. Comparing spectra $\mathrm{A}$ and $\mathrm{B}$, we find only marginally higher ionisation parameters for components $2-4$ in spectrum A.

Figure 4 shows the change in $C$-statistics for spectra $\mathrm{A}$ and $\mathrm{D}$ when the ionisation parameter for each component is separately varied. Step size is 0.1 in $\log \xi$ and at each step the goodness of fit is calculated. It is clear from this figure that no significant changes can be detected for components 1,3 and 4 . We discuss the variability of component 2 in more detail later.

In Fig. 5 we present the model absorption spectrum for each component, labelling the deepest lines. It thus allows the reader to see which component contributes to which line. The continuum was renormalised to ease identification.

\subsection{Broad emission lines}

Besides absorption lines, strong emission features are present in the four spectra. We chose two different methods for fitting this excess emission: a model with broadened emission lines and a model in which the absorbers only partially cover the continuum. We first describe the model with broadened emission lines. As was the case for fitting the absorber, we first fitted spectrum $\mathrm{C}$ and then used its best fit values as a starting point to fit the other spectra (see Table 1 for best fit parameter values). The presence of a broad O VIII Ly $\alpha$ emission feature around $19 \AA$ is illustrated in Fig. 6, which shows a detail of the spectra B and C including the best fit model with and without relativistically broadened O VIII Ly $\alpha$. Figure 7 shows the model emission spectrum for spectra B and C. Excess emission with at least a $1 \AA$ width, due to the O VII triplet near $22 \AA$ is present in spectra A, C and D. In addition, there is significant excess emission in spectra $\mathrm{C}$ and $\mathrm{D}$ near $34 \AA$, the region of the $1 \mathrm{~s}-2 \mathrm{p}$ transition in C VI.

We modelled the excess emission at $19 \AA$ (see Fig. 7) with a relativistically broadened line, following Ogle et al. (2004) and Branduardi-Raymont et al. (2001). The model used is a narrow emission line convolved with the relativistic disc line profile of Laor (1991). The inner $\left(r_{1}\right)$ and outer $\left(r_{2}\right)$ disc radii were fixed in our models to the default values $\left(r_{1}=1.234 G M / c^{2}\right.$ and $r_{2}=$ $\left.400 G M / c^{2}\right)$. The rest-frame wavelength of the line was frozen to $18.969 \AA$, the wavelength of O VIII Ly $\alpha$.

We have tried fits with different disc inclinations $i$, but in all fits the inclination converged to $48^{\circ}$, within a few degrees. This value is consistent with the one found by Ogle et al. (2004). The disc inclination is not expected to change significantly over a couple of years. For that reason, and in order to reduce the number of free parameters, we have kept the inclination frozen to that value in all presented fits. The disc emissivity slope $q$ (with $R^{-q}, R$ the distance to the black hole) was allowed to vary, however.

In addition to a relativistic O VIII Ly $\alpha$ line, we added a Gaussian-shaped broad O VIII line Ly $\alpha$ line (FWHM $0.45 \AA$ ) to our model for spectrum $C$ and D. Further broad lines corresponding to the $\mathrm{O}$ VII triplet and $\mathrm{C}$ VI Ly $\alpha$ line were also needed. For C VI Ly $\alpha$, we have frozen the wavelength to the rest wavelength of $33.736 \AA$. For O VII we kept the wavelength as a free parameter: the line is a blend of the O VII triplet, and the relative 



Fig. 2. Flux (in units of photons $\mathrm{m}^{-2} \mathrm{~s}^{-1} \AA^{-1}$ ) and our best spectral model. All spectra are binned by a factor of 2 for clarity of display. Note the different continuum levels and shapes between the spectra, especially around the upturn at $\sim 16 \AA$. In spectrum D C VI and weaker C V RRCs at $25.43 \AA$ and $31.80 \AA$ respectively, are observed. 
Table 3. The best fit parameters for the RRCs in spectrum D. The velocity refers to the rest frame of NGC 4051.

\begin{tabular}{lc}
\hline \hline Parameter & Value \\
\hline$k T(\mathrm{eV})$ & $5 \pm 2$ \\
$\mathrm{C} \mathrm{V}{ }^{a}$ & $210 \pm 110$ \\
$\mathrm{C} \mathrm{VI}$ & $390 \pm 80$ \\
$\mathrm{~N} \mathrm{VI}^{a}$ & $40 \pm 60$ \\
$\mathrm{~N} \mathrm{VII}^{a}$ & $30 \pm 30$ \\
$\mathrm{O}$ VII & \\
$\mathrm{O}$ VIII & \\
velocity $^{a}\left(\mathrm{~km} \mathrm{~s}^{-1}\right)$ & $0 \pm 20$ \\
\hline
\end{tabular}

${ }^{a}$ Emission measures in $10^{64} \mathrm{~m}^{-3}$, e.g., $\mathrm{X}=\mathrm{C}$ VII produces the $\mathrm{RRC}$ observed for C VI.

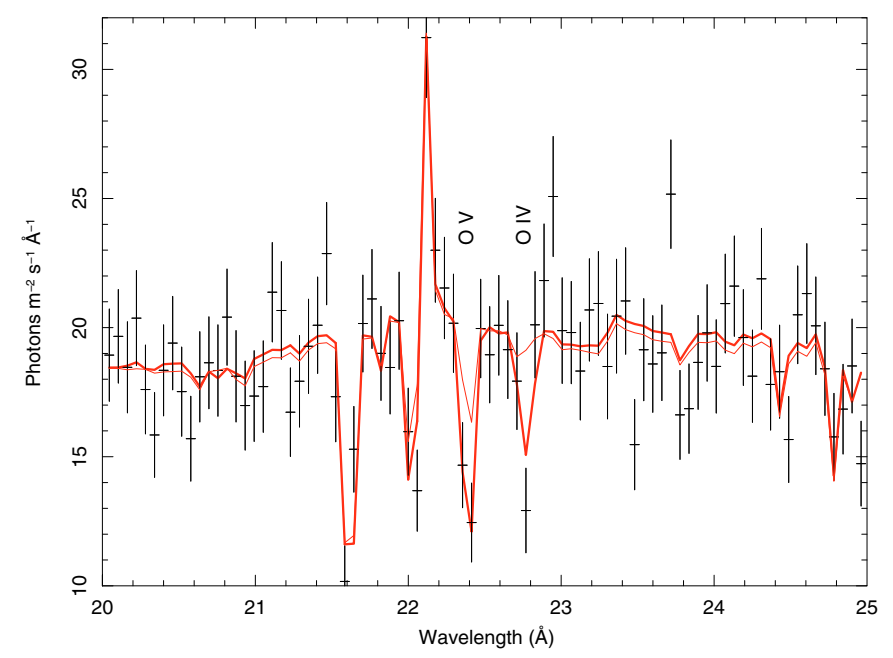

Fig. 3. Detail of spectrum C fitted with 3 warm absorber components (thin line) and 4 warm absorber components (thick line). Binning as in Fig. 2. The best fit 3 component model does under-predict the depth of the $\mathrm{O} v$ and $\mathrm{O} I \mathrm{~V}$ lines.

contributions of these lines depend on the physical conditions in the source. Such broadened emission lines were first detected in NGC 5548 by Kaastra et al. (2002), and confirmed for that source by Steenbrugge et al. (2005), and were detected in this source by Ogle et al. (2004).

\subsection{Partial covering absorption model}

As an alternative to fitting extra emission lines, we fitted the spectra assuming that the absorber does not fully cover the continuum emission. Considering that the excess emission is most significantly detected in spectrum B and C, we first fitted these two spectra (see Table 1 and Fig. 6). In this fit there are no broad emission lines fitted. We fixed the covering factor for the 3 or 4 detected absorption components (spectrum B and C, respectively) to 0.75 , and then refitted the continuum parameters (power law and modified black body) and the hydrogen column density and ionization parameter of the detected absorber components. Afterwards we also allowed the covering factor for each absorption component free to vary in the fit.

For spectrum $\mathrm{C}$ the best fit covering factors for three of the four absorption components is 1 . For warm absorber component 2 the best fit covering factor is $0.7 \pm 0.13$, with an increased hydrogen column density of $0.63 \times 10^{25} \mathrm{~m}^{-2}$ (compared with $0.29 \times 10^{25} \mathrm{~m}^{-2}$ for a covering factor of 1 ). Forcing all covering factors to 1 increases $\Delta C$ by 17 . However, the best fit with
Table 4. The best fit parameters for a three-absorber-component model for spectrum C. Components 1-3 correspond to components $2-4$ in Tables 1 and 2.

\begin{tabular}{lc}
\hline \hline Parameter & Value \\
\hline$\bullet$ power law & \multicolumn{2}{c}{} \\
Flux $(2-10 \mathrm{keV})^{a}$ & $2.86 \pm 0.04$ \\
Photon index $\Gamma$ & $2.21 \pm 0.02$ \\
$\bullet$ modified black body & \\
flux $(0.2-10 \mathrm{keV})^{a}$ & $1.31 \pm 0.14$ \\
$k T(\mathrm{eV})$ & $140 \pm 4$ \\
\hline Absorption components \\
$\bullet$ warm absorber 1 \\
$N_{\mathrm{H}}\left(10^{25} \mathrm{~m}^{-2}\right)$ & $0.35 \pm 0.14$ \\
$\log \xi\left(10^{-9} \mathrm{~W}\right)$ & $0.73 \pm 0.06$ \\
$v_{\text {turb }}\left(\mathrm{km} \mathrm{s}^{-1}\right)$ & $147 \pm 21$ \\
$v_{\text {out }}\left(\mathrm{km} \mathrm{s}^{-1}\right)$ & $-213 \pm 27$ \\
$\bullet$ warm absorber 2 & \\
$N_{\mathrm{H}}\left(10^{25} \mathrm{~m}^{-2}\right)$ & $0.72 \pm 0.3$ \\
$\log \xi\left(10^{-9} \mathrm{~W} \mathrm{~m}\right)$ & $2.27 \pm 0.06$ \\
$v_{\text {turb }}\left(\mathrm{km} \mathrm{s}^{-1}\right)$ & $100 \pm 28$ \\
$v_{\text {out }}\left(\mathrm{km} \mathrm{s}^{-1}\right)$ & $-548 \pm 45$ \\
$\bullet$ warm absorber 3 & \\
$N_{\mathrm{H}}\left(10^{25} \mathrm{~m}^{-2}\right)$ & $23 \pm 7.5$ \\
$\log \xi\left(10^{-9} \mathrm{~W} \mathrm{~m}\right)$ & $3.13 \pm 0.04$ \\
$v_{\text {turb }}\left(\mathrm{km} \mathrm{s}^{-1}\right)$ & $8 \pm 8$ \\
$v_{\text {out }}\left(\mathrm{km} \mathrm{s}^{-1}\right)$ & $-4530 \pm 80$ \\
\hline
\end{tabular}

${ }^{a}$ Absorption-corrected flux in $10^{-14} \mathrm{~W} \mathrm{~m}^{-2}$.

partial covering increases $\Delta C$ by 70 for a decrease of 7 in degrees of freedom, compared with the best fit model with broad emission lines (4 extra free parameters in the partial absorption fit compared to 11 in the model with broad emission lines). A comparison between the best fit with broad emission lines and a fit with all absorbers having a covering factor of 0.75 can be seen in the bottom panel of Fig. 8. Note that the continuum long-ward of $\sim 24 \AA$ is consistently over-estimated in the fit assuming that the absorber only covers part of the continuum emission.

For spectrum B we followed the same procedure, but here the best fit for the covering factors of the three absorption components detected are 1 , even if we redid the fitting starting from a covering factor of only 0.25 . The best fit without broad emission lines increases $\Delta C$ by 21 and is shown in Fig. 6. Forcing the three absorbers to have a partial covering factor of 0.75 worsens the fit by a further 35 in $\Delta C$. This last fit is compared with the best fit with broad emission lines in the upper panel of Fig. 8. Note that again the continuum is over-estimated at long wavelengths, furthermore the model assuming partial covering cannot reproduce the steep rise at $16 \AA$.

\subsection{Narrow emission lines}

The O VII forbidden line at $22.10 \AA$ is detected with at least $4 \sigma$ significance in each individual spectrum. There is no indication for significant flux variability or velocity broadening for this line.

In spectrum $\mathrm{D}$ a strong, $\sim 5 \sigma$ significant, RRC from $\mathrm{C}$ VI (rest wavelength $25.30 \AA$ ) and a $\sim 2 \sigma$ significant RRC of $\mathrm{C} \mathrm{V}$ (rest wavelength $31.62 \AA$ ) are present (see Fig. 2 and Table 3 ). In addition, we have determined limits to the strength of other expected RRCs of oxygen and nitrogen ions (see Table 3). The temperature of the recombining photoionised gas is low but not well determined $(T=5 \pm 2 \mathrm{eV})$. In our final fit we fix the temperature to $5 \mathrm{eV}$, in order to better assess the significance of this component. 

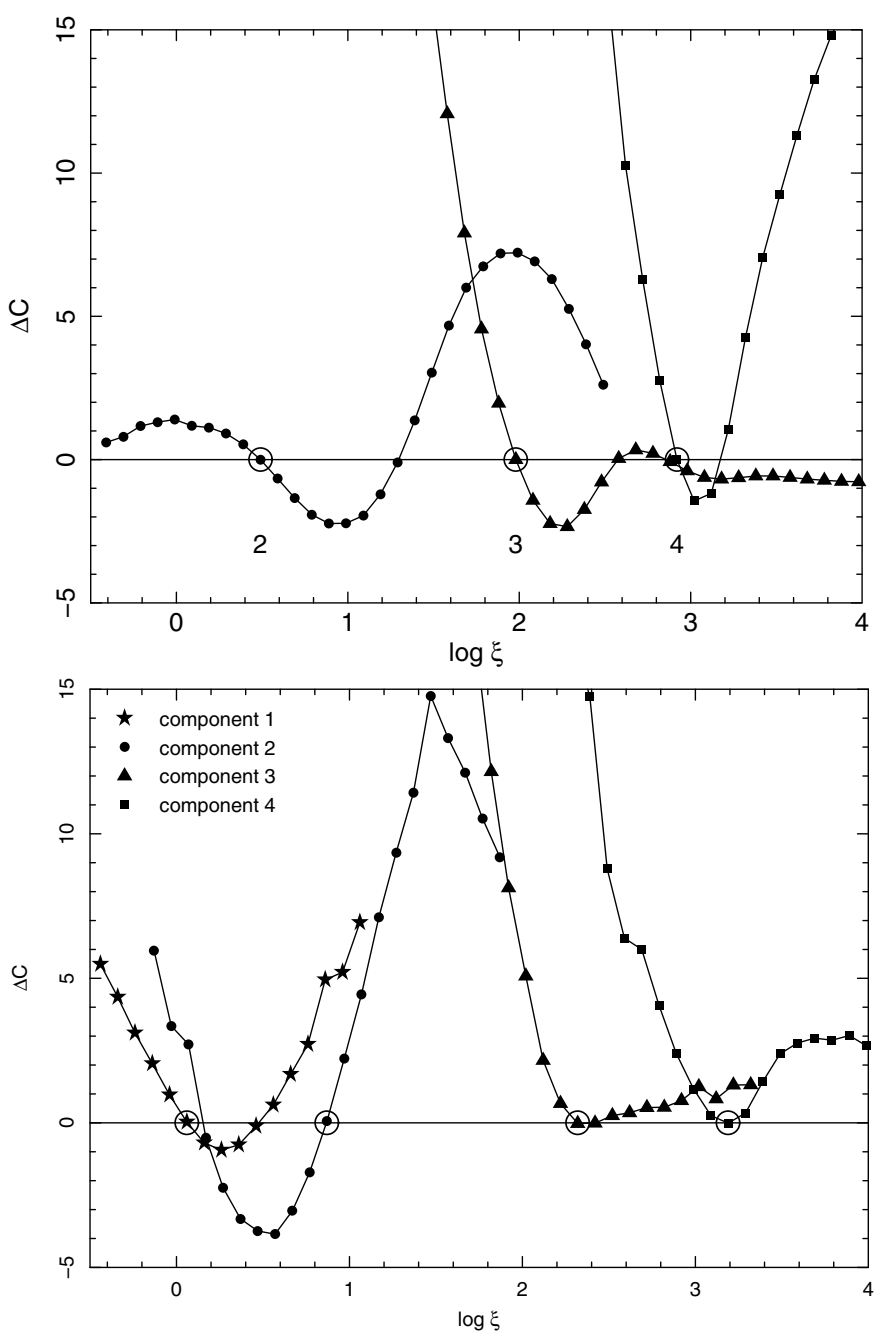

Fig. 4. Change $(\Delta C)$ in $C$-statistic for spectrum $\mathrm{A}$ (top panel) and spectrum D (bottom panel) when the ionisation parameter $\xi$ of each component is varied. Open circles indicate the reference values of each component (for spectrum $\mathrm{D}$, the value of $\xi$ is the one determined from spectrum $\mathrm{C}$; for spectrum $\mathrm{A}$, the value of $\xi$ is the one determined from spectrum B).

There is a strong correlation between the adopted temperature and the strength of the emission measure. The RRCs show a redshift with respect to the rest frame of the galaxy of $\sim 1300 \mathrm{~km} \mathrm{~s}^{-1}$.

\section{Discussion}

\subsection{Continuum}

The uniqueness of the transition between part $\mathrm{C}$ and $\mathrm{D}$ is that the rather abrupt flux change is preceded and followed by long periods of relative quiescence. This allows us to study the response of the continuum, the broad and narrow emission lines, and the warm absorber to this sudden flux drop. We first describe the difference in the continuum. Figure 9 shows the difference in overall continuum profile for spectrum $\mathrm{C}$ and $\mathrm{D}$.

Between spectrum C and D the temperature measured for the modified black body significantly decreases, and the power-law slope becomes softer. The temperature remained constant and the power-law slope softened between spectrum A and B. For Seyfert 1 galaxies, normally, the spectral slope hardens with decreasing luminosity with little or no change in the black body component. Pounds et al. (2004) studying two EPIC spectra

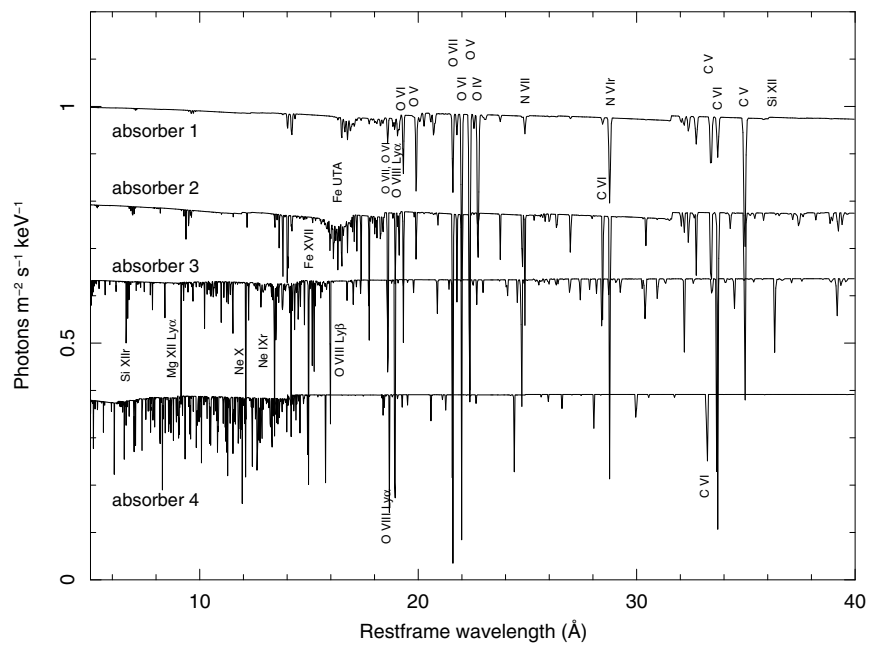

Fig. 5. The model absorption spectra for each of the 4 different absorption components as detected in spectrum $\mathrm{C}$. The continuum was modelled to be flat and was renormalised per component.

taken in a high and a low state of NGC 4051 found that the higher temperature black body component disappeared in the low state. The authors found that the power-law slope did not vary between the high and low state, which is different from our results.

Changes in the warm absorber properties could mimic a temperature change of the modified black body component. To ensure that in the spectra there really is a temperature change we did the following tests. We refitted spectrum D, but freezing the temperature of the modified black body to the best fit value of spectrum C. In the fit the column density and ionisation parameter of the four absorption components were left free to vary. The fit thus obtained was substantially worse (increasing $C$-statistics by 102), and the fitted ionisation parameters were very similar to those obtained in the original fit of spectrum D. Afterwards, we lowered the ionisation parameter of the only significantly detected warm absorber component in spectrum D by a (linear) factor 5. This decrease matches the decrease in luminosity observed. Leaving the ionisation parameters of the absorber and the temperature of the modified black body free in the fit,we again derive our original fit parameters given in Table 1.

Ogle et al. (2004) studying the XMM-Newton RGS spectrum, find that a multi-temperature disc model is a poor fit to the soft excess, and that instead it is well fitted by the relativistically broadened O VIII line series. We tested this model on our highest signal-to-noise spectrum, C, by adding to the O VIII Ly $\alpha$ line also the $\operatorname{Ly} \beta$, Ly $\gamma$ and the $\mathrm{RRC}$ emission lines relativistically broadened. As none of these emission features are obvious from a visual inspection, we fixed the relative normalisation of the different lines to the $\operatorname{Ly} \alpha$ component: 0.25 for $\operatorname{Ly} \beta, 0.1$ for $\operatorname{Ly} \gamma$ and 0.21 for the RRC. The parameters $q$ and $i$ were free in the fit, but the same for the four different emission lines. The fit obtained was significantly worse (increasing $C$-statistics by 195 ) for the same number of parameters than a fit with a modified black body.

We conclude that there is emission from a modified black body present in the LETGS spectrum, and that the temperature of this component drops with decreasing luminosity between spectrum $\mathrm{C}$ and $\mathrm{D}$.

\subsection{Relativistically broadened O VIII Ly $\alpha$ line}

The relativistically broadened O VIII Ly $\alpha$ line which explains part of the RGS soft excess (Ogle et al. 2004) is significantly 


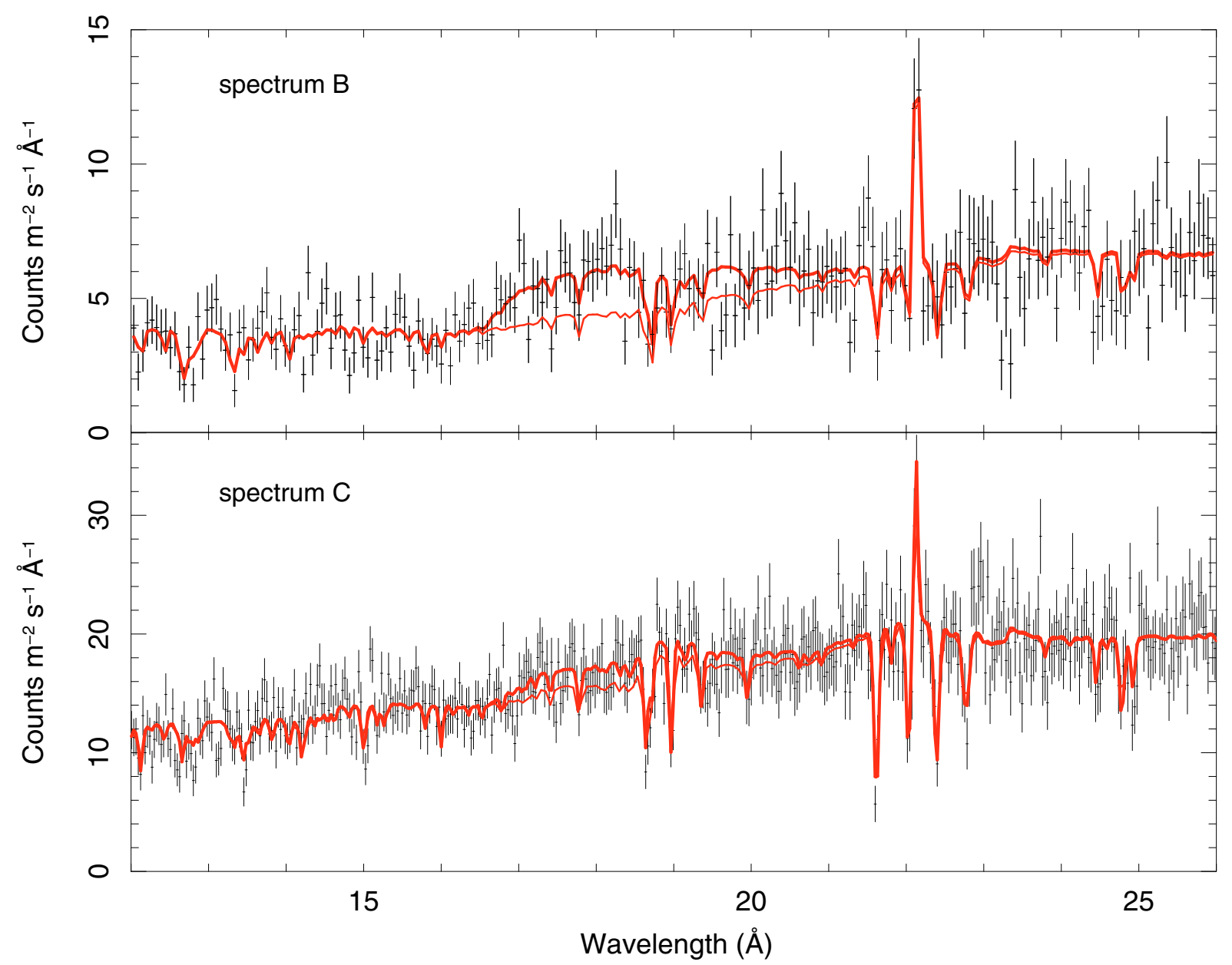

Fig. 6. Detail of spectra B and C with the best fit model (thick line) and the best fit model without the O VIII Ly $\alpha$ relativistically broadened emission line (thin line).

detected in all spectra (see Table 1). The measured line fluxes do not follow the power law or modified black body continuum flux changes. The emissivity $q$, assuming $R^{-q}$, is similar to the value $q_{2}=5.0$ measured by Ogle et al. (2004) for spectrum B, but not for spectrum $\mathrm{C}$. We note that for spectrum $\mathrm{A}$ and $\mathrm{D}$ the emissivity was frozen to the value for spectrum $\mathrm{B}$ and $\mathrm{C}$ respectively. Ogle et al. (2004) in detail discusses the steep emissivity derived as well as the physical parameters in the accretion disc derived from the line profile and the lack of C VI Ly $\alpha$ relativistic line. The latter places a lower limit on the ionisation parameter. Summarized, the relativistic O VIII Ly $\alpha$ is emitted between 1.6 and 6 gravitational radii, $\xi>3.4$ and $n_{\mathrm{e}}<10^{23} \mathrm{~m}^{-3}$.

The inclination angle for all spectra was consistent with $48^{\circ}$; very similar to the inclination measured by Ogle et al. (2004). The inclination angle for the narrow line region (NLR) as derived by Christopoulou et al. (1997) from narrow band [O III] imaging and long-slit spectroscopy using the Manchester Echelle Spectrograph is about $50^{\circ}$. This indicates that the narrow line region, and the gas producing the relativistically broadened emission line lie along the same line of sight. The narrow line region is therefore possibly an extension of the gas producing the relativistically broadened emission lines. If this is the case, then the gas forming the relativistically broadened emission line is outflowing, similar to the absorption components. From the kinematics, Christopoulou et al. (1997) conclude that the imaged [O III] gas is outflowing. The inclination angle of the host galaxy is at medium angles (Möllenhoff \& Heidt 2001), thus gas observed from the relativistic and narrow emission lines are closely aligned with the host galaxy.

Relativistic line profiles in the soft X-ray band were first reported by Branduardi-Raymont et al. (2001) in the narrow lines Seyfert 1 galaxies MCG -6-30-15 and Mrk 766. Interestingly, the relativistically broadened O VIII, N VII and C VI Ly $\alpha$ lines have only been convincingly detected in narrow line Seyfert 1 galaxies. For MCG -6-30-15 and Mrk 766 the disc line parameters derived from the soft X-ray band and the Fe-K line are in good agreement. The XMM-Newton observation of NGC 4051 that showed a strong relativistic O VIII line (Ogle et al. 2004) did not show a significant relativistic Fe-K line (Pounds et al. 2004). Unfortunately the LETGS is not sensitive enough in the Fe-K band to directly compare the disc geometry as derived from iron and oxygen lines. For the physical implications of the relativistic lines in NGC 4051 we refer to the discussion by Ogle et al. (2004).

\subsection{Broad emission lines}

The broad O VII line, centred on the forbidden line, is detected in spectrum $\mathrm{C}$, but the line is weaker or absent in the other spectra. Ogle et al. (2004) detected a broadened O VII emission line with a full width half maximum $(F W H M)$ of $11000 \pm$ $3000 \mathrm{~km} \mathrm{~s}^{-1}$. This is consistent with the $F W H M$ we derive: $24000 \pm 6000 \mathrm{~km} \mathrm{~s}^{-1}$. Both values are rather larger than the FWHM determined from the $\mathrm{H} \beta$ and $\mathrm{He}$ II broad lines measured 

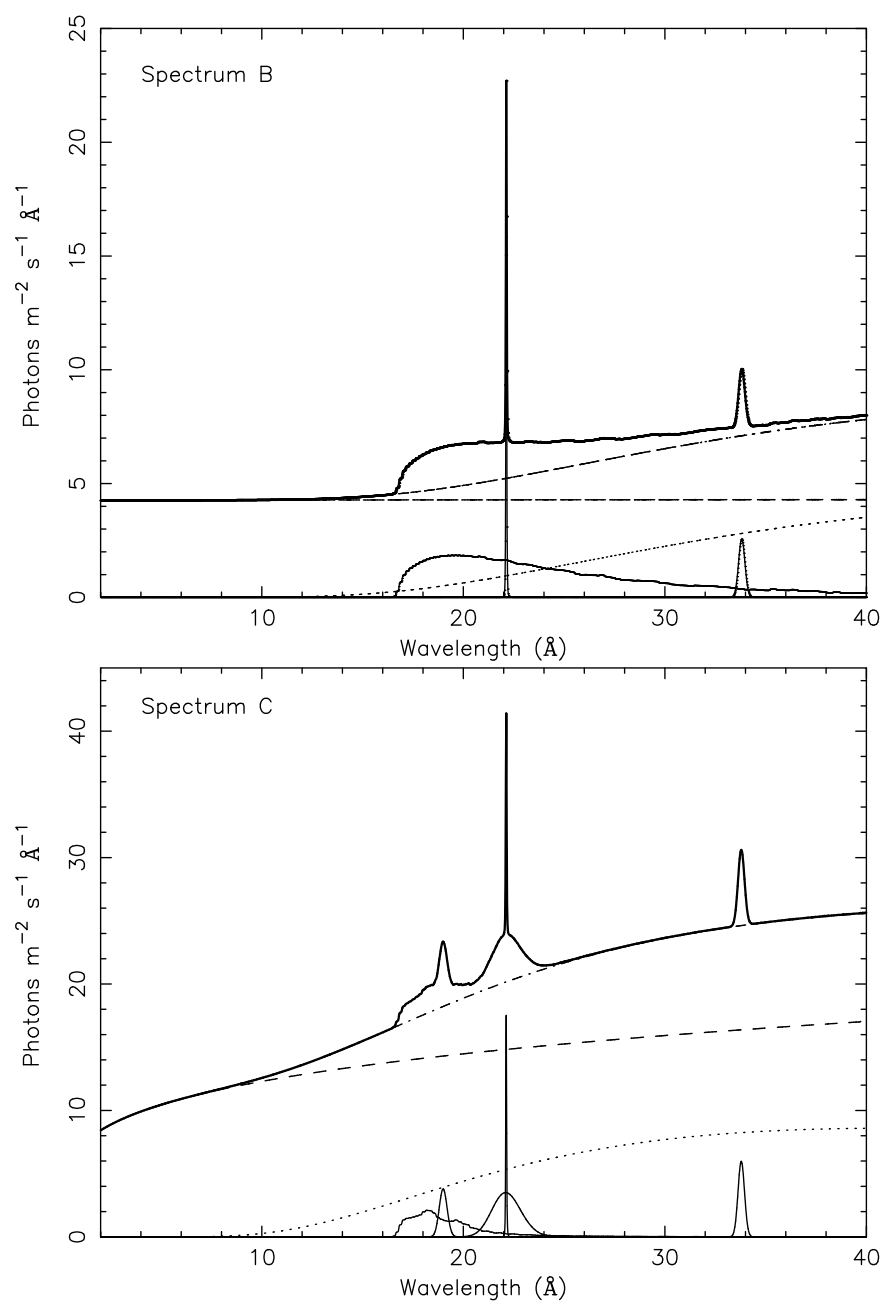

Fig. 7. Model emission spectra for spectrum B and C, without Galactic absorption and the warm absorber components. Dashed line: power law continuum; dotted line: modified black body continuum; dash-dotted line: sum of power law and modified black body continuum; thin solid lines: relativistic, broad and narrow emission line contributions; thick solid line: total unabsorbed emission spectrum.

in the UV (Peterson et al. 2000). If the broad line region (BLR) has a stratified ionisation structure, then it is expected that the higher ionised O VII line will have a broader width than either the $\mathrm{H} \beta$ or lowly ionised He II. At $1.7 \AA$ the width of the broadened O VII line exceeds the separation $(0.5 \AA)$ between the forbidden and resonance line. The broadened resonance (and intercombination) lines contribute to the flux and hence the width measured.

We also detect a broadened C VI line with a width of $3200 \pm$ $1000 \mathrm{~km} \mathrm{~s}^{-1}$, which is smaller by a factor of $\sim 7$ than the $\mathrm{O}$ VII line. This value is fairly similar to the $F W H M$ derived for the $\mathrm{H} \beta$ line $(1100 \pm 190$, Peterson et al. 2000) and the width measured for this line in the RGS spectrum $\left(1200 \mathrm{~km} \mathrm{~s}^{-1}\right.$, Ogle et al. 2004). The smaller width is consistent in terms of a stratified BLR model, with the fact that C VI is formed at lower ionisation parameters.

In spectrum $\mathrm{C}$ on top of the relativistically broadened $\mathrm{O}$ VIII Ly $\alpha$ emission line there is a broadened O VIII Ly $\alpha$ emission line.

Costantini et al. (2007) studying Mrk 279 did detect broadened emission lines with better statistics than is the case for NGC 4051. The authors conclude that the broad X-ray emission lines originate from a stratified BLR. The above findings for NGC 4051 are consistent with this conclusion.

\subsection{Narrow emission features}

During our observations we detect various narrow emission features in the spectra. The narrow forbidden O VII line was significantly detected in all spectra, and all flux values are consistent with each other to within $1 \sigma$ (see Table 1 ). This is consistent with the picture that the $\mathrm{O}$ VII forbidden line is formed at the narrow line region further out. The width of this line (it is unresolved), precludes it being formed as part of the outflow.

In spectrum D we detect the C VI RRC and the C V RRC. In the other three spectra these features are not detected. The shapes and centroids of individual RRCs are determined by the temperature of the recombining gas, the emission measure of the relevant ion, and by the motion (velocity broadening) of the recombining gas. We derived the temperature and emission measure for the RRCs assuming no velocity broadening. We have investigated the effect of broadening by including a velocity broadening component in our RRC spectral model. On the basis of the goodness of fit we exclude that the turbulent velocity in the recombining gas is greater than $3000 \mathrm{~km} \mathrm{~s}^{-1}$. Both the temperature and emission measure are within the error bars the same for models with and without a velocity broadening of $3000 \mathrm{~km} \mathrm{~s}^{-1}$.

If we interpret the maximum velocity broadening as due to Keplerian velocity at the location where the RRC is formed and assume a black hole mass of $M=3 \times 10^{5} M_{\odot}$ (McHardy et al. 2004) we obtain a minimum distance for the recombining gas of $R \sim 4 \times 10^{12} \mathrm{~m}$, or $\sim 4500$ Schwarzschild radii.

From the fitted temperature of the RRC of $T=5 \pm 2 \mathrm{eV}$, we derive an ionisation parameter $\log \xi \simeq 1$.6. With the ionising luminosity of the source $\left(L=2.8 \times 10^{35} \mathrm{~W}\right)$, the minimum distance derived above and the definition of $\xi$, we obtain a maximum density of $4 \times 10^{17} \mathrm{~m}^{-3}$. It is possible to estimate a lower limit to the volume of the RRC emitting source using the observed ionic emission measure of the C VI RRC $\left(3.9 \times 10^{66} \mathrm{~m}^{-3}\right)$, a solar carbon abundance (Anders \& Grevesse 1989) and a fraction of $60 \%$ in the form of C VII (appropriate for $\log \xi=1.6$ ). We find an emitting volume of $V=10^{35} \mathrm{~m}^{3}$. Note that the volume might be larger than the emitting volume if the filling factor is smaller than unity. We should note that CLOUDY (Ferland et al. 2004) version 95.06 calculates the size of the clouds to be of order $10^{11} \mathrm{~m}$, thus a filling factor of less than unity is rather unlikely. We equate this volume to $\Omega R^{2} \Delta R$ where $\Delta R$ the characteristic thickness of the emitter in the radial direction and $\Omega$ the solid angle sustained by the emitter. Dividing the above formula by $R^{3}$ and substituting the values for the volume and minimum distance we find

$\Omega \frac{\Delta R}{R}=0.002$.

Taking for the distance a value of $4 \times 10^{13} \mathrm{~m}$, instead of $4 \times$ $10^{12} \mathrm{~m}$, we derive a value of 0.02 .

Another constraint follows from the column density $N_{\mathrm{H}}$ through the emitting region. This can be written as $N_{\mathrm{H}}=n \Delta R \leq$ $n R(0.002 / \Omega)$ (assuming the filling factor is unity, see Eq. (1)). Taking the minimum radius and maximum density we can get an indication of the column density: $N_{\mathrm{H}} \sim 3 \times 10^{26}(4 \pi / \Omega) \mathrm{m}^{-2}$.

A possible origin of the RRCs is that the gas in one (or more) of the warm absorber components is recombining. To test this hypothesis, we compare the properties of the recombining gas with that of the warm absorber components. The large estimated column density for the RRC is only derived for absorption 


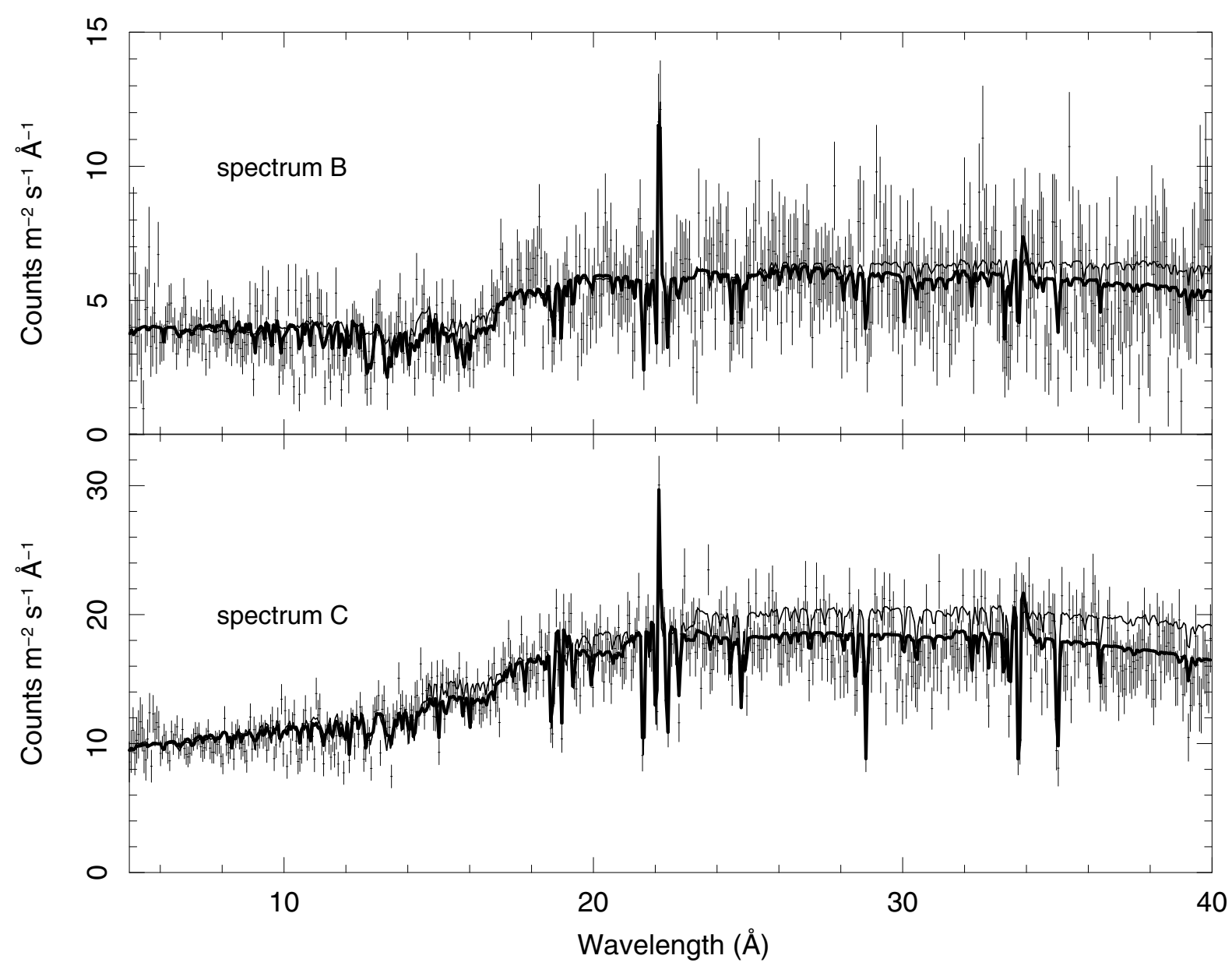

Fig. 8. Top: detail of the spectrum for the best fit (thick line) model with broad emission lines and the best fit (thin line) assuming the absorber has a covering factor of .75 for spectrum B. Bottom: same as above but for spectrum C.

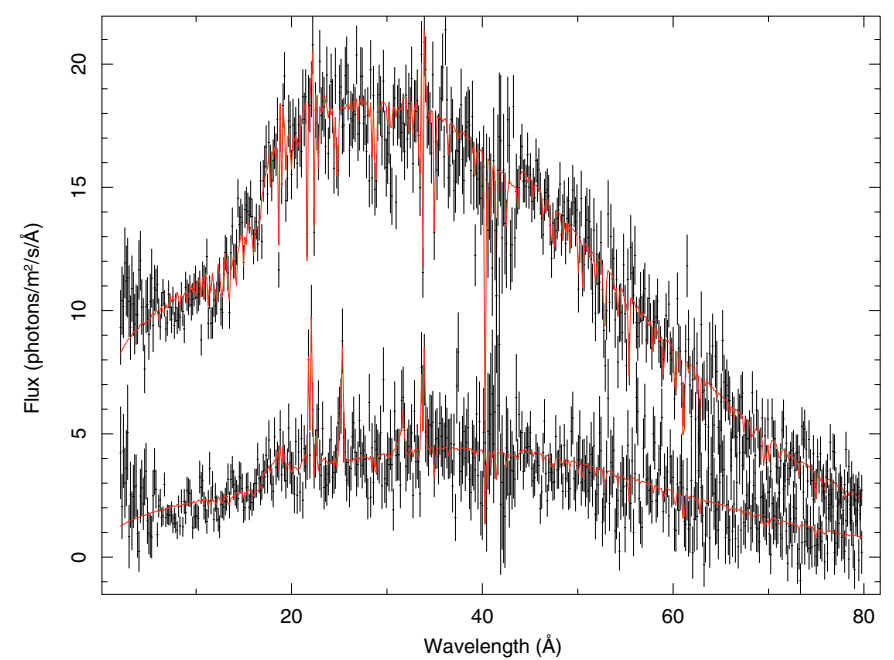

Fig. 9. The spectrum for part $\mathrm{C}$ and $\mathrm{D}$, clearly showing the difference in continuum shape. The difference is due to a lower flux in the powerlaw component and a lower temperature for the modified black body component in spectrum D.

component 4. However for this component the ionisation parameter is substantially higher, making a connection between this absorption component and the gas producing the RRCs unlikely.
Component 2 has a similar ionisation parameter to the RRCs but its column density in spectrum B and C is substantially smaller. Thus this connection is also unlikely. Alternatively the RRCs could originate in the thin, ionised skin of the accretion disc. The width of the $\mathrm{C}$ VI broad emission line is similar to the maximum broadening of the RRCs. Also the ionization parameters are similar.

For $\log \xi \simeq 1.6$ we expect that most of the oxygen gas will recombine into $\mathrm{O}$ VII, thus we expect a strong O VII RRC at 16.77 $\AA$. This RRC is not observed, and we can place an upper limit for the emission measure of $0.6 \times 10^{66} \mathrm{~m}^{-3}$. It is possible that, the many absorption lines due to different iron ions mask the presence of the $\mathrm{O}$ VII RRC, or that the temperature is lower than $5 \mathrm{eV}$.

Ogle et al. (2004) detected the C VI RRC in the high flux state RGS spectrum; Pounds et al. (2004) detected in a different low state RGS spectrum the C VI and O VII RRCs. Both authors derive a temperature of $3 \mathrm{eV}$, consistent with our derived temperature of $5 \pm 2 \mathrm{eV}$.

\subsection{The ionised absorber}

In our spectra, we have found evidence for the presence of four warm absorber components (Table 1). The absorption components are discussed in detail below. 


\subsubsection{Warm absorber component 1}

The least ionised component, which has a low outflow velocity and the smallest hydrogen column density, is not detected in spectrum A or B, due to the lower signal to noise in these spectra. The strongest lines are due to O IV, O V and C V.

\subsubsection{Warm absorber component 2}

This component has a similarly low outflow velocity as component 1 , but with a higher ionisation parameter, $\log \xi=0.87$. The strongest lines of this component are the deep $1 \mathrm{~s}-2 \mathrm{p}$ absorption lines of O VII, O VI and O V. This is the only component for which we measure a decrease by a factor of two in the ionisation parameter between spectrum C and D (see Table 1, Fig. 4). This component, perhaps partly together with our component 1 , corresponds to the low ionisation component found by Krongold et al. (2007). However, they find a column density that is twice as large and a somewhat higher outflow velocity of $-500 \mathrm{~km} \mathrm{~s}^{-1}$.

\subsubsection{Warm absorber component 3}

With an outflow velocity of $-580 \mathrm{~km} \mathrm{~s}^{-1}$, component 3 has the largest opacity. The dominant imprints are the $\operatorname{Ly} \alpha$ transitions of hydrogenic oxygen, nitrogen and carbon. The outflow velocity and ionisation state are similar to one of the two absorber components reported by Collinge et al. (2001). McKernan et al. (2007) analysing the same spectrum found three components, two of which are consistent with our component 2 and 3 . Note that the observed Doppler velocity (sum of the $+700 \mathrm{~km} \mathrm{~s}^{-1}$ heliocentric velocity of NGC 4051, Verheijen \& Sancisi (2001), and the intrinsic outflow velocity of $\sim-600 \mathrm{~km} \mathrm{~s}^{-1}$ ) is only $+100 \mathrm{~km} \mathrm{~s}^{-1}$, and less than $2 \sigma$ different from 0 . Therefore, this absorption could be blended with absorption from our Galaxy or the local group. Pounds et al. (2004) analysed the high state RGS spectrum; and detected an absorber with $\log \xi=2.7$ and an outflow velocity of $\sim-600 \mathrm{~km} \mathrm{~s}^{-1}$, which can probably be associated with this component. We associate this component with the high ionisation component of Krongold et al. (2007), for which these authors derive a distance of $1-2 \times 10^{13} \mathrm{~m}$ based on time variability; but see Sect. 5.5.6 below.

\subsubsection{Warm absorber component 4}

The last absorber component has the highest ionisation parameter, hydrogen column density and outflow velocity. The C VI and $\mathrm{N}$ VII Ly $\alpha$ lines are the deepest detected. The O VIII Ly $\alpha$ blends with and broadens the less blueshifted secondary O VII line. The outflow velocity of $-4670 \mathrm{~km} \mathrm{~s}^{-1}$ was first reported by van der Meer et al. (2003). This is one of the highest outflow velocities derived from high resolution spectra of Seyfert galaxies, that we are aware of. Collinge et al. (2001) observed for the highest ionised component in a Chandra HETG spectrum of NGC 4051, an outflow velocity component of $-2340 \mathrm{~km} \mathrm{~s}^{-1}$, which is not detected in our spectra. The high velocity component that we detect, and the one reported by Collinge et al. (2001), seem to be the only ionised components detected in the X-rays without a UV absorber at the same outflow velocity. In many AGN the most highly ionised X-ray absorber should not produce any UV absorption lines, however usually a UV absorber with the same outflow velocity as the X-ray absorber is detected. This does not seem to be the case for the $-4670 \mathrm{~km} \mathrm{~s}^{-1}$ component we detect. Both the $-2340 \mathrm{~km} \mathrm{~s}^{-1}$ and the $-4670 \mathrm{~km} \mathrm{~s}^{-1}$ components are highly ionised and therefore detectable with the HETG, the LETG and the RGS. Ogle et al. (2004) do not detect these highly ionised components in the RGS spectrum. Considering that neither we nor Ogle et al. (2004) detect the $-2340 \mathrm{~km} \mathrm{~s}^{-1}$ component, and that the $-4670 \mathrm{~km} \mathrm{~s}^{-1}$ component is not present in the earlier Chandra HETG and RGS data, these high velocity absorbers either have a variable column density or are transient.

\subsubsection{Comparison between the absorber components}

The derived hydrogen column density and ionisation parameters for our three absorbers with an ionisation parameter $\log \xi<3$ are in agreement with those derived from an RGS observation, in a similar flux state, and given in a figure by Ogle et al. (2004). We confirm, using the parameters measured for spectrum $\mathrm{C}$, that there is an increase in hydrogen column density with increasing ionisation parameter, as observed in this source by Ogle et al. (2004) and in NGC 5548 by Steenbrugge et al. (2005).

It is also possible to constrain the opening angle of the outflow. From the ionising luminosity $\left(L=2.8 \times 10^{35} \mathrm{~W}\right)$ and the definition of $\xi$, we get $n r^{2}=L / \xi$. As we measure the outflow velocity $v$, we know that the mass outflow rate per steradian is $m_{\mathrm{p}} n r^{2} v$. We list this value for each of the absorber components in Table 4, based on our result for spectrum C. Assuming a typical accretion efficiency of $10 \%$, the observed luminosity of NGC 4051 corresponds to an accretion rate of $5 \times 10^{-4} M_{\odot} \mathrm{yr}^{-1}$. Assuming the mass outflow through the wind is smaller than the accretion onto the black hole, we can put upper limits to the solid angle sustained by the outflow. This assumption seems reasonable as the energy needed to accelerate the outflow must come from the infalling gas. These upper limits are small, typically less than $10^{-2}$ to $10^{-5}$ sr. Using this same formalism, similar to somewhat larger opening angles were derived for the outflow in NGC 5548 (Steenbrugge et al. 2005).

\subsubsection{Time variability of the warm absorber}

Our spectral modelling of the warm absorber components is based upon models for photoionisation balance. Whenever the ionising flux of the source changes, the ionisation balance should adjust to the new situation, and the ionisation parameter $\xi$ should change. The speed at which this happens depends on the density $n$ of the absorbing material. The recombination timescale $\tau_{i}$ for a given ion $i$ is inverse proportional to the gas density. It is given by Bottorff et al. (2000) to be

$\tau_{i} n=\left[\alpha_{i}\left(\frac{f_{i+1}}{f_{i}}-\frac{\alpha_{i-1}}{\alpha_{i}}\right)\right]^{-1}$

where $f_{i}$ is the relative concentration of ion $i$ of a given element, and $\alpha_{i}$ is the recombination rate of ion $i$, and in general depends only on the temperature $T$ of the gas. This formula does take into account the cascade into and out of ion $i$. Furthermore, a negative value for the recombination time indicates that the ion is destroyed by recombination to the ion $i-1$. For a given photoionisation equilibrium, the temperature is a unique function of the ionisation parameter $\xi$, and the ion concentrations are also known as a function of $\xi$. Therefore, given a value for $\xi$, the right hand side of (Eq. (2)) can be evaluated.

The largest flux change occurs between spectrum C and D. The hard 2-10 keV power-law flux drops suddenly by a factor of $6.1(\log p=-0.79$, where we define $p$ as the flux ratio of spectrum D to spectrum C). The soft flux (taken here and later as the 0.2-10 keV modified black body flux from Table 1) decreases 
Table 5. Observed and calculated parameters for the four warm absorber components of spectrum $\mathrm{C}$.

\begin{tabular}{lcccc}
\hline \hline Component & 1 & 2 & 3 & 4 \\
\hline $\log \xi$ & 0.07 & 0.87 & 2.32 & 3.19 \\
$\log T(\mathrm{~K})$ & 4.21 & 4.33 & 5.14 & 5.83 \\
$n r^{2}\left(\mathrm{~m}^{-1}\right)$ & $2.4 \times 10^{44}$ & $3.8 \times 10^{43}$ & $1.3 \times 10^{42}$ & $1.8 \times 10^{41}$ \\
$v\left(\mathrm{~km} \mathrm{~s}^{-1}\right)$ & -210 & -200 & -580 & -4670 \\
$m_{\mathrm{p}} n r^{2} v\left(M_{\odot} \mathrm{yr}^{-1}\right)$ & 1.3 & 0.20 & 0.021 & 0.022 \\
$\Omega(\mathrm{sr})$ & $<3.8 \times 10^{-4}<2.5 \times 10^{-3}<2.4 \times 10^{-2}<2.2 \times 10^{-2}$ \\
\hline most abundant & \multicolumn{5}{c}{} & \\
oxygen ion $i$ & $\mathrm{O} \mathrm{V}$ & $\mathrm{O}$ VII & O VIII & O VIII \\
$\tau_{i} n\left(\mathrm{~s} \mathrm{~m}^{-3}\right)$ & $5.3 \times 10^{16}$ & $8.8 \times 10^{16}$ & $8.5 \times 10^{16}$ & $7.4 \times 10^{15}$ \\
For $\tau_{i}<20 \mathrm{ks}$ or $\tau_{i}>20 \mathrm{ks}:$ & & & \\
$\operatorname{limit}$ on $r(\mathrm{~m})$ & $>9 \times 10^{15}$ & $<3 \times 10^{15}$ & $>5 \times 10^{14}$ & $>7 \times 10^{14}$ \\
For $\tau_{i}<570$ days: & & & \\
limit on $r(\mathrm{~m})$ & - & $<2 \times 10^{17}$ & $<3 \times 10^{16}$ & $<4 \times 10^{16}$ \\
\hline
\end{tabular}

by a factor of $5.4(\log p=-0.73)$. Thus, if the density in one of the components is high, we would expect $\log \xi$ to decrease by about 0.73 , and if the density is low, we expect no change between spectrum $\mathrm{C}$ and $\mathrm{D}$. As the low state of spectrum D lasts about $20 \mathrm{ks}$ and our data have insufficient $\mathrm{S} / \mathrm{N}$ to resolve spectrum $\mathrm{D}$ further in time, this gives the limit on the recombination timescale.

Indeed in components 1, 3 and 4 we see no significant change in $\log \xi$, and definitely no change as large as -0.73 (see Table 1 ), indicating that the recombination timescale is longer than $20 \mathrm{ks}$. This then gives the lower limits to the distance of these components from the central source as listed in Table 5. In the table the product $n r^{2}$ is calculated from $L / \xi$. The product of recombination timescale and density $\tau_{i} n$ is calculated from (Eq. (2)), using the total recombination rates of Nahar (1999). Limits on $r$ are calculated from $r=\sqrt{\left(n r^{2}\right)\left(\tau_{i}\right) /\left(\tau_{i} n\right)}$. The lower limit for component 3 , namely $5 \times 10^{14} \mathrm{~m}$ (19 light days), disagrees with the distance of $0.5-1$ light days derived by Krongold et al. (2007) for this component.

Krongold et al. (2007) studied the variability using the relatively low-resolution EPIC data. Some possible reasons for the disagreement are given below. Krongold et al. (2007) do not allow the continuum shape (i.e. photon index and temperature) to vary, while at least in our data the shape of the continuum significantly changes (see Fig. 9). Further, Krongold et al. (2007) do not include a relativistic O VIII Ly $\alpha$ line although in the total and high state spectra their best fit model (see their Figs. 2 and 3) is consistently below the observed continuum between 17 and $19.2 \AA$. Similarly, the continuum is above their model between 20 and $21.8 \AA$, where we fit a broadened O VII line. Failure to fit the local continuum correctly will result in too low or too high column densities derived from the line depths.

Krongold et al. (2007) included only two absorption components and have problem fitting some of the oxygen lines. Therefore, their model cannot explain the O V absorption line at $22.4 \AA$, which is easily observed in their published RGS spectrum. Furthermore, the depth of the O VII line is overestimated in the high state, and underestimated it in the low state. Oxygen lines are diagnostically important because of their large oscillator strength (i.e. strong lines) and the rather large range in ionisation parameters they span. One can thus determine (over this ionisation range) the ionisation structure without having to worry about abundance effects. Finally, long term differences between our observations and those of Krongold et al. (2007), could explain the differences found in ionisation variability behaviour.
Krongold et al. (2007) claim to detect variability in the ionisation parameters on timescales of $2-3 \mathrm{ks}$, leading to a much larger derived density and thus closer location of the absorber.

In our component 2 , we see a decrease of a factor of 2 $(\Delta \log \xi=-0.35 \pm 0.16)$, suggesting that the recombination time is indeed $20 \mathrm{ks}$ or smaller. This now gives an upper limit to the distance of $3 \times 10^{15} \mathrm{~m}$ or 110 light days (see Table 5). This is higher than, but consistent with the upper limit of 3.5 light days for the low ionisation component of Krongold et al. (2007). That the change in $\log \xi$ is not the expected value of -0.73 may have two reasons, which we cannot be distinguished in our present data. The recombination timescale might be close to our upper limit, so that the gas is still recombining at the end of interval D. Alternatively, the flux changes in the invisible EUV band might be smaller, and these may be more important for the low ionisation gas than the X-ray continuum (the dominant oxygen ions for component 2 are $\mathrm{O}$ VI and $\mathrm{OV}$ ).

Next we investigate the long term variability, by comparing spectrum $B$ to spectrum $C$ in a similar way as we did above for spectra D and C. The soft and hard flux of spectrum B have $\log p=-0.48$ and $\log p=-0.33$, respectively. Taking a typical value of -0.4 , we see that all visible components in spectrum $B$ (components 2-4) are in agreement with such a change in $\log \xi$ $(\Delta \log \xi=-0.38 \pm 0.22,-0.34 \pm 0.26$ and $-0.27 \pm 0.19)$. We conclude that the recombination timescale for these components is likely faster than 570 days, the interval between both observations. This gives interesting upper limits to the distance of components 3 and 4 (see Table 4 ) of about 1 pc. We should note that components $2-4$ could coexist in the same location, but that component 1 and 2 can not coexist in the same location, due to the lack of variability in component 1 , even over 570 days.

Finally, we have an independent short term comparison by comparing spectrum A to spectrum B, although our discriminating power is smaller here, because of the smaller flux jump $(\log p=-0.13$ and -0.21 for the soft and hard band, respectively). The error bars on the ionisation parameter for components 3 and 4 preclude a definite conclusion here; for component 2 , we see an increase in spectrum A of $\Delta \log \xi=+0.46 \pm$ 0.32 , which favours the no change scenario, but does not exclude a small response compatible with $\log p=-0.13$. We also note that, of all our four spectra, the flux during spectrum A shows the largest variations within the given interval (Fig. 1), so the establishment of any (quasi)-equilibrium during that interval may be questioned.

\section{Summary}

In this study we have investigated the spectral properties of NGC 4051, observed by Chandra-LETGS on two occasions for a total of $180 \mathrm{ks}$. Due to the flux variations within each observation, we split each observation into 2 spectra, to be fit separately. In the highest signal-to-noise spectrum, $\mathrm{C}$, we detect four absorption components. Our main results are:

- The continuum parameters do change between the different observations, the power-law slope becomes softer with time. The temperature of the modified black body increases, to first order, with increasing luminosity.

- Between spectra C and D, when the flux dropped by a factor of 5 , absorbers 1,3 and 4 show no evidence for a change in ionisation parameter; however, for absorber 2 the ionisation parameter does decrease.

- Our limits on the variability of the absorber suggest that component 2 is located within $0.1 \mathrm{pc}$, and components 3 
and 4 are located somewhere in the range $0.02-1$ pc. For component 1 , we only have a lower limit of $0.3 \mathrm{pc}$. These distances are different from those derived by Krongold et al. (2007).

- We detect one of the highest outflow velocities $\left(-4670 \mathrm{~km} \mathrm{~s}^{-1}\right)$ for an ionised absorber observed in the soft X-ray band. We do not confirm the $-2340 \mathrm{~km} \mathrm{~s}^{-1}$ absorber detected by Collinge et al. (2001), indicating that these high outflow velocity absorbers have variable column densities or are transient.

- The RRCs of C VI and C V are detected in spectrum D, after a drop in flux by factor 5 . These are unlikely due to recombining gas from one of the absorber components, but are possibly from the accretion disc.

- Broad emission lines of the O VII triplet and O VIII Ly $\alpha$ and C VI Ly $\alpha$ are detected. These lines show a simple Gaussian profile, suggesting an origin in the BLR.

- We detect a relativistically broadened O VIII Ly $\alpha$ line, similar to the findings by Ogle et al. (2004).

Acknowledgements. K.C.S. thanks St John's College for a fellowship. SRON is supported financially by NWO, the Netherlands Organization for Scientific Research.

\section{References}

Anders, E., \& Grevesse, N., 1989, Cheochim. Cosmochim. Acta, 53, 197 Baldwin, J., Ferland, G., Korsita, K., \& Verner, D. 1995, ApJ, 455, L119 Branduardi-Raymont, G., Sako, M., Kahn, S. M., et al. 2001, A\&A, 365, L140 Brinkman, A. C., Gunsing, A. C., Kaastra, J. S., et al. 2000, ApJ, 530, L111 Bottorff, M. C., Korista, K. T., \& Shlosman, I. 2000, ApJ, 537, 134

Christopoulou, P. E., Holloway, A. J., Steffen, W., et al. 1997, MNRAS, 284, 385 Collinge, M. J., Brandt, W. N., Kaspi, S., et al. 2001, ApJ, 557, 2
Costantini, E., Kaastra, J. S., Arav, N., et al. 2007, A\&A, 461, 121 Elvis, M., Wilkes, B. J., \& Lockman, F. J. 1989, AJ, 97, 777

Elvis, M., Krongold, Y., Nicastro, F., Brickhouse, N., \& Binette, L. 2005, in The X-ray Universe 2005, ESA SP-604, 539

Ferland, G. J., Korista, K. T., Verner, D. A., et al. 2004, PASP, 110, 761

Gabel, J. R., Kraemer, S. B., Crenshaw, D. M., et al. 2005, ApJ, 631, 741

Hubble, E. P. 1926, ApJ, 64, 231

Iwasawa, K., Fabian, A. C., Reynolds, C. S., et al. 1996, MNRAS, 282, 1038

Kaastra, J. S., \& Barr, P. 1989, A\&A, 226, 59

Kaastra, J. S., Mewe, R., \& Nieuwenhuijzen, H. 1996, in Frontiers Science Ser. 15, UV and X-ray Spectroscopy of Astrophysical and Laboratory Plasmas, ed. K. Yamashita, \& T. Watanabe (Tokyo: Univ. Ac. Press), 411

Kaastra, J. S., Steenbrugge, K. C., Raassen, A. J. J., et al. 2002, A\&A, 386, 427 Kaspi, S., Brandt, W. N., Collinge, M. J., Elvis, M., \& Reynolds, C. S. 2004, AJ, 127,2631

Krongold, Y., Nicastro, F., Elvis, M., et al. 2007, ApJ, 659, 1022

Laor, A. 1991, ApJ, 376, 90

Lawrence, A., Watson, M. G., Pounds, K. A., \& Elvis, M. 1987, Nature, 325, 694

McHardy, I. M., Papadakis, I. E., Uttley, P., Mason, K. O., \& Page, M. J. 2004, MNRAS, 348, 783

McKernan, B., Yaqoob, T., \& Reynolds, C. S. 2007, MNRAS, 379, 1359

van der Meer, R. L. J., Kaastra, J. S., Steenbrugge, K. C., \& Komossa, S. 2003, ASP Conf. Ser., 290, 133

Möllenhoff, C., \& Heidt, J. 2001, A\&A, 368, 16

Nahar, S. N. 1999, ApJS, 120, 131

Nandra, K., \& Pounds, K. A. 1994, MNRAS, 268, 405

Netzer, H., Kaspi, S., Behar, E., et al. 2003, ApJ, 599, 933

Ogle, P. M., Mason, K. O., Page, M. J., et al. 2004, ApJ, 606, 151

Peterson, B. M., McHardy, I. M., Wilkes, B. J., et al. 2000, ApJ, 542, 161

Pounds, K. A., Reeves, J. N., King, A. R., \& Page, K. L. 2004, MNRAS, 350, 10

Seyfert, C. K. 1943, ApJ, 97, 28

Steenbrugge, K. C., Kaastra, J. S., Crenshaw, D. M., et al. 2005, A\&A, 434, 569

Tully, R. B., \& Pierce, M. J. 2000, ApJ, 533, 744

Tully, R. B., Verheijen, M. A. W., Pierce, M. J., Huang, J.-S., \& Wainscoat, R. J. 1996, AJ, 112, 2471

Uttley, P., Fruscione, A., McHardy, I., \& Lamer, G. 2003, ApJ, 595, 656

Verheijen, M. A. W., \& Sancisi, R. 2001, A\&A, 370, 765 